\title{
Transition towards a new global change science: Requirements for methodologies, methods, data and knowledge
}

\author{
Claudia Pahl-Wostl ${ }^{a, *}$, Carlo Giupponi ${ }^{b}$, Keith Richards ${ }^{c}$, Claudia Binder ${ }^{d}$, \\ Alex de Sherbinin ${ }^{e}$, Detlef Sprinz ${ }^{f}$, Theo Toonen ${ }^{g}$, Caroline van Bers ${ }^{h}$ \\ ${ }^{a}$ Institute of Environmental Systems Research, University of Osnabrueck, Barbarastr. 12, 49069 Osnabrueck, Germany \\ ${ }^{\mathrm{b}}$ University of Venice, Department of Economics, Cannaregio 873, I-30121 Venice, Italy \\ ${ }^{\mathrm{c}}$ Department of Geography, Cambridge University, United Kingdom \\ d Ludwig-Maximilians-University, Munich, Germany \\ e Center for International Earth Science Information Network, The Earth Institute, Columbia University, Palisades, NY 10964, USA \\ ${ }^{\mathrm{f}}$ Potsdam Institute for Climate Impact Research, Transdisciplinary Concepts \& Methods, 14412 Potsdam, Germany \\ g Delft Technical University, The Netherlands \\ ${ }^{\mathrm{h}}$ Seeconsult, Osnabrueck, Germany
}

\section{A R T I C L E I N F O}

Keywords:

Global change

Innovation

Methodologies

Methods

Data and knowledge

\begin{abstract}
A B S T R A C T
Dealing with the challenges of global change requires a transition not only in society but also in the scientific community. Despite continued claims for more inter-disciplinary approaches, progress to date has been slow. This paper elaborates on the need for innovation in methodologies and knowledge, on the one hand, and methods and data, on the other, to build the foundations for dealing with the challenges from global change. Three questions related to the nature of global change, the dynamics of sustainability transitions and the role of human agency guide analyses on the state of the art, barriers for innovation and need for action. The analyses build on literature reviews, expert workshops and surveys which were conducted under the umbrella of RESCUE, a foresight activity funded by the European Science Foundation. The major recommendations focus on integrating environmental and human dimensions, bridging scales, data and knowledge for global change research and overcoming structural constraints to make global change research more policy relevant.
\end{abstract}

(C) 2012 Published by Elsevier Ltd.
1. Introduction

Global change poses unprecedented challenges to scientific and policy communities, of the kind that cannot be tackled using existing conceptual frameworks and disciplinary approaches and methods. Two challenges seem to be particularly relevant and persistent: the integration of natural and social sciences ${ }^{1}$ and the production of societally relevant knowledge.

Both the natural and the social sciences have sought to address global change challenges, the former through the

\footnotetext{
* Corresponding author. Tel.: +49 541969 2536; fax: +49 5419692599.

E-mail addresses: cpahlwos@uni-osnabrueck.de, pahl@usf.uni-osnabrueck.de (C. Pahl-Wostl), cgiupponi@unive.it (C. Giupponi), ksr10@cam.ac.uk (K. Richards), Claudia.Binder@geographie.uni-muenchen.de (C. Binder), adesherbinin@ciesin.columbia.edu (A. de Sherbinin), dsp@pik-potsdam.de (D. Sprinz), T.A.J.Toonen@tudelft.nl (T. Toonen), cvanbers@seeconsult.org (C. van Bers).

${ }^{1}$ Social sciences is used here as an umbrella term to refer to all fields of academic scholarship that deal with human society, such as philosophy, anthropology, economics, political science, and sociology. 1462-9011/\$ - see front matter (C) 2012 Published by Elsevier Ltd.
} http://dx.doi.org/10.1016/j.envsci.2012.11.009 
development of Earth System Science (Schellnhuber, 1999) and the latter mainly through critical analysis of globalisation processes (e.g. Amin and Thrift, 1994). However, these alternative conceptualisations have not been integrated, despite recognition that new forms of inter-disciplinary knowledge creation, and new forms of inquiry, are needed. In fact, although the Amsterdam Declaration ${ }^{2}$ of the Global Change Programmes (2001) expressed the need to move towards a more integrated perspective, the research agenda of these programmes continues to be framed and dominated by the natural sciences (Reid et al., 2009). A survey conducted in 2011 by the International Human Dimension Programme (IHDP), in collaboration with UNESCO and the International Social Science Council (ISSC), explored the current status of engagement of social science scholars in Global Environmental Change (GEC) research, and collected more than 1200 questionnaires from multi-disciplinary experts around the world. ${ }^{3}$ There was general agreement on the need to address human dimensions of GEC more in the future, with priority consideration of issues such as equity, governance, economic policies, and social and cultural transitions. ${ }^{4}$

However, an effective integration of societal concerns into scientific practice may require more fundamental changes in the nature of scientific enquiry, and a move towards truly inter-disciplinary research, and also involving external stakeholders in the research process. Gibbons et al. (1994) distinguish conventional, "Mode 1 " forms of science from a "Mode 2" form in which knowledge production is guided by using values mutually and reflexively constructed by a heterogeneous set of practitioners and experts working together (see also Irwin's "citizen science"; 1995). Kates et al. (2001), following the Amsterdam declaration ${ }^{2}$ outlined a research programme for sustainability science that would focus on the dynamic interactions between nature and society, analyse the resilience of social-ecological systems, and bridge science and practice to support societal transitions toward sustainability. These developments can all be interpreted as the first steps towards a transition in scientific research. However, cross-cutting initiatives in research and capacity building promoted at the Amsterdam conference, including joint projects on carbon, food, health and water, have taken time to get under way, suggesting that there continue to be difficulties in conducting inter-disciplinary research. Funding challenges have contributed to this delay, but as Webster (2007) notes, the critical social sciences may be suspicious of co-option and capture by natural science agendas, and unwilling simply to act as a medium through which science can be rendered more acceptable to various publics. Instead, social scientists want to have their understanding integrated at the earliest stages of project formula-

\footnotetext{
2 The Amsterdam Declaration on Global Change was adopted during the first joint congress of the four global change programmes "Challenges of a Changing Earth: Global Change Open Science Conference Amsterdam, The Netherlands (www.essp.org/ index.php?id=41).

${ }^{3}$ The survey report can be downloaded at http://www.ihdp. unu.edu/file/get/9091.

${ }^{4}$ In accordance with Rotmans et al. (2001), we define transitions as transformation processes in which society - or part of it changes in a fundamental way.
}

tion, so that more radical transformations of knowledge production can be envisaged (Hackmann and St. Clair, 2012).

These difficulties can be interpreted using the concept of different levels, or stages, of social learning process that describe both intellectual and societal transitions (PahlWostl, 2009). Here, "single-loop learning" refers to incremental improvement of action strategies, without questioning underlying assumptions; "double-loop learning" then refers to a revisiting of assumptions (e.g. about cause-effect relationships) within a value-normative framework. However, it is only through "triple-loop learning" that underlying values, beliefs and world views begin to be reconsidered, and assumptions and world views are challenged. From this perspective, one can argue that the global change research community has entered the phase of double-loop learning, in which there is a reframing of the dominant research paradigm, to which the "human dimensions" community has made a significant contribution. The first signs of tripleloop learning, which requires structural change, are now emerging. Such structural change includes the adoption of new, shared norms, together with changes in actor-network structures, and in the roles of actor groups (Pahl-Wostl, 2009). This is an emergent process where emphasis needs to be given to methodologies, modes of inquiry and sharing of knowledge.

Much has been written on the problems and research questions to be addressed in global change research, but far less attention has been devoted to the requirements for methodologies, methods, knowledge and data to address these challenges. Given the recognition that barriers for innovation reside in deeply entrenched procedures and practices, we explicitly chose to broaden our concern to include "methodologies and knowledge" as well as "methods and data". Sometimes these terms are used synonymously, but we consider it important to expand the terminology, and to be precise about the different meanings of the terms.

Thus, a "methodology" sets the framework for combining modes of inquiry and methods, and forms a set of organizing principles, following the logic underlying a particular area of study (or science). A "method", however, is a specific information generation practice; measuring devices generate data, methods generate information, and methodologies generate knowledge. These distinctions largely follow the DIKW (Data-Information-Knowledge-Wisdom) model (Rowley, 2007). "Data" are symbols, such as the numbers produced by a temperature-measuring device, whereas "information" places data in relation to some meaning that makes them useful (e.g., impacts of July temperature on the yield of a certain crop). "Knowledge" is information embedded in a context of interpretation (e.g., the ability to make temperature-sensitive crop choices based on experience or expert knowledge). Knowledge embraces framed experience, contextual information and grounded intuition (Davenport and Prusack, 1998; Wallace, 2007), and is embedded in routines, practices and norms that may not always be explicit. These definitions indicate that simply to address "methods and data" is to assume a particular mode of knowledge production, and accordingly, constrains our enquiry and our interest in exploring how science can support transitions towards sustainable development in a changing world. 
This paper is based on deliberations of Working Group 3 on Requirements for Research Methodologies and Data of the European Science Foundation Forward Look on "Responses to Environmental and Societal Challenges for our Unstable Earth (RESCUE)". 5 It elaborates on requirements for methodologies and knowledge, in the context of the global change research considered necessary to meet future challenges. It starts from the premise that a lack of appropriate methodologies and knowledge may well be a bottleneck in dealing with the major challenges facing global change research. We first summarize guiding questions and the materials and methods used to develop and substantiate our argument (Section 2). Section 3 presents the current state and promising developments, together with gaps, barriers and the needs for action for the three challenges identified below. Discussion and conclusion (Sections 4 and 5) then highlight the key insights derived from our analyses regarding key actions and provide a roadmap to help us overcome barriers.

\section{Guiding principles and methods used in developing this paper}

\subsection{Global change research-major challenges}

In 2009, the International Council for Science (ICSU) and the International Social Science Council (ISSC) together launched a broadly based visioning process on global change research for global sustainability. This identified five related grand challenges: Forecasting, Observation, Thresholds, Responses, and Innovation (ICSU, 2010). It also emphasized the need for a transition from research dominated by the natural sciences to research involving the social sciences and humanities; and from purely disciplinary research to a balance of disciplinary, and inter- and trans-disciplinary ${ }^{6}$ research, involving systemic attribution of a central role to the human dimension, This call has been reiterated in the recent ISSC report, Transformative Cornerstones of Social Science Research for Global Change, which emphasizes the central role of social science knowledge in contributing to change in institutions, regulatory systems, financial regimes, as well as individual attitudes and practices, lifestyles, policies and power relations (Hackmann and St. Clair, 2012, p. 16). The final goal of this transition to new modes of research is to support the broader transition towards sustainability, and societal transformations will necessarily be part of that transition.

We have developed three guiding questions drawing on issues addressed by the ICSU grand challenges, these being:

\footnotetext{
${ }^{5}$ In this special issue other companion papers deal with Collaboration between the natural, social and human sciences in global change studies, Towards a 'revolution' in education and capacity building, and Interface between science and policy, communication and outreach (the other Working Group themes).

${ }^{6}$ In this paper we make a clear distinction between inter- and trans-disciplinarity, with the first resulting from the combination and integration of distinct disciplinary approaches within the scientific domain, and the latter going beyond the boundaries of scientific disciplines by involving both scientists and stakeholders in a cooperative approach to solve relevant societal issues, and specifically those related to sustainable development.
}

1. What are the characteristics of global environmental challenges that make them not easily amenable to policy solutions, and how can innovation in knowledge, methodologies, methods and data, support identification and implementation of sustainable policy solutions?

2. How do we manage transitions to sustainable resource governance and management regimes, while at the same time respecting good governance principles? What data and methods and which kind of innovation in knowledge and methodologies are required to understand transition processes and support their "management"?

3. What is the role of human agency (as consumers, change agents and political entrepreneurs) in developing adaptive responses to global change in social-ecological systems? What are knowledge, methodologies, methods and data needed to analyse the role of individual behaviour, and the factors at both the individual and the societal levels that influence this behaviour; and what are the drivers of and barriers to change?

We argue that these questions capture the key themes and concerns identified in the ICSU visioning exercise, and are therefore well suited to an enquiry into the requirements for methodologies and knowledge.

\subsection{Sources, methods and definitions}

The working group undertook a thorough assessment of the state of the art, and of the latest developments and innovations in the knowledge, methodologies, methods and data required by GEC research. To address the guiding questions identified above, the group itself used a range of approaches including a literature review, an online survey, expert interviews, an expert workshop, and the development of position papers. While more limited in scope than the ISSC's GEC Design Project (Hackmann and St. Clair, 2012), the methods were quite similar.

\subsubsection{On-line survey}

An online survey on knowledge gaps and major structural constraints to innovation was implemented in February 2010 in collaboration with The Integrated Assessment Society (TIAS). Questions included in the survey focused on how to improve representation of the human dimension in global change research, how to develop more policy-relevant integrated GEC models, how to analyse the dynamics of multi-level and complex governance systems for natural resources, and how best to develop shared databases and protocols. The survey was aimed at the GEC scientific research community and the policy community using the research results. A profile of the respondents is in Table 1. The majority of the academic respondents were senior scientists based in Europe and North America working on interdisciplinary research topics. Responses were processed by sub-groups during a RESCUE workshop in March 2010, each taking a different set of questions and synthesising the responses.

\subsubsection{Expert interviews}

Working group members conducted 24 interviews in April and May 2010 to test the inferences from the survey, and to identify 
Table 1 - RESCUE Survey on GEC Methodologies and Data: Respondent Profiles.

\begin{tabular}{|c|c|}
\hline Total no. of respondents & 26 (20 men, 6 women) \\
\hline Country where based & $\begin{array}{l}9 \text { US, } 4 \text { Canada, } 4 \text { UK, } 2 \text { Austria, } 2 \text { Australia/NZ, } 1 \text { Brazil, } 1 \text { Bahrain, } 1 \text { Hungary, } 1 \text { Sub-Saharan Africa, } \\
1 \text { Netherlands }\end{array}$ \\
\hline Respondents holding PhDs. & 22 \\
\hline Affiliations & 17 academic/research institutes, 5 NGOs or IFIs, 2 regional government, 2 consulting firms \\
\hline Position/level & $\begin{array}{l}1 \text { doctoral candidate, } 2 \text { post-doctoral researchers, } 16 \text { senior researchers or senior specialists, } 1 \text { senior } \\
\text { lecturer, } 2 \text { assistant professors, } 2 \text { associate professors, } 1 \text { consultant, } 1 \text { professor }\end{array}$ \\
\hline Field of Study/Research & $\begin{array}{l}\text { Social sciences (e.g. political science, international development, public health) and natural sciences } \\
\text { (e.g., chemical kinetics, biogeochemical modelling), and combinations of the two (i.e., political ecology, } \\
\text { geography, natural resources management) }\end{array}$ \\
\hline Expertise & $\begin{array}{l}\text { Environmental informatics, environmental/climate modelling, science and policy of global change, } \\
\text { environmental decision-making in climate and adaptation, ecological footprint policy, sustainability } \\
\text { indicators and reporting, and scenario analysis }\end{array}$ \\
\hline
\end{tabular}

gaps, barriers, and new developments. The three main questions being addressed by the RESCUE Working Group were each supplemented by sub-questions developed on the basis of the survey outcomes. Interviewees included 7 scientists, 1 data developer, and representatives of funding agencies (6), a bank (1), international organisations (2), and an industry association (1). Interviewers worked to a protocol/ guidance document, and respondents were provided with a background paper on the activities of the Working group. The questions are listed in Table 2.

\subsubsection{Expert workshop}

In June 2010, a two-day expert workshop was organised at the Institute for Environment and Sustainability at the Joint Research Council in Ispra, Italy, bringing together a small group representing the scientific community, NGOs, and funding agencies. Together with working group members, the purpose was to validate the results of the survey and the interviews, to further enhance insights into gaps and barriers, to identify new opportunities and priorities for innovation in GEC research and to derive recommendations. Break-out groups addressed each of the three key questions of the working group and developed a set of recommendations for both long-term and short-term activities.

\subsubsection{Position papers}

Brief position papers were solicited from Working Group members and external experts on specific themes (general GEC, water, climate change, agriculture/food, energy, spatial planning); the three key questions; and challenges and best practice. These papers provide raw material for the Working Group report and this paper. In total 18 topics for these briefing notes were defined and 15 were completed.

\section{Findings and inferences}

Taken together, the outcomes of the five approaches outlined above have provided a rich array of insights and recommendations on the needs for innovation and the barriers to be overcome in GEC methods and data, as well as methodologies and knowledge. This section on the findings follows the logic of the three guiding questions. It draws especially on the interviews and on outcomes of the expert workshop, since

\section{Table 2 - Framework of questions used in RESCUE Interviews.}

A. Characteristics of Global Environmental Challenges

A1. Are there characteristics of global environmental challenges that make them not easily amenable to policy solutions? If yes, which (up to 5 characteristics)? If no, why not (up to 5 reasons)?

A2. Can current practice/innovation in data and methods support identification and implementation of longer-term policy solutions? If yes, how? If no, why not?

A3. What are the barriers to innovation in data and methods?

B. Human Agency

B1. What is your institutional role in developing adaptive responses to global change in human-technology-environment systems? OR (if more appropriate to the respondent's situation)

What is the role of citizens and consumers in adaptive and mitigation responses to global change in human- technology-environment systems?

B2. What innovations are needed in developing data and methods for the study of environmentally relevant human behaviour?

B3. What do you consider to be the barriers to these innovations?

C. Transitions/Change Management

C1. How can your organisation contribute to managing transitions/change to integrated and sustainable resource governance and management regimes while at the same time respecting good governance principles (esp. accountability, legitimacy, transparency, effectiveness and efficiency)?

C2. What innovations in data and methods are required to understand transition/change processes and support their management?

C3. What are the barriers to these innovations? 
they focused on in-depth analysis of knowledge gaps and major structural constraints, and in particular on how to overcome them. However, reference is also made to results from the online survey and interview responses.

\subsection{Characteristics of global environmental challenges}

The first guiding question (see Section 2.1) seeks to characterise global environmental challenges, which are commonly also long-term, and have been defined as "public policy issues that last at least one human generation, exhibit deep uncertainty exacerbated by the depth of time, and engender public goods aspects both at the stage of problem generation as well as at the response stage" (Sprinz, 2009, p. 2). This points to the multi-decadal time scales, structural complexity, and uncertainty inherent in global environmental challenges, and to the potentially wide range of stakeholders, who may have different value systems, frame problems in different ways, and differ in their views on both the analysis of, and solutions to, those problems. The pervasive complexity of global environmental challenges means that they defy conventional problem-solving approaches relying on reductionism.

\subsubsection{The nature of the global challenge}

The outcomes of the multiple methods deployed in our enquiry confirm and supplement the characteristics of global change and policy, identifying as critical: mismatches in space and time scales, the inter- and trans-disciplinary character of the research required to support policy, and the multi-level uncertainties associated with global change.

Spatial scale. While climate change may be global in scale, its manifestations are spatially complex, and this inhibits policy formulation. There is no system of global governance that can manage environmental issues successfully, but as Underdal (2008) reminds us, international institutions are not necessarily the most effective means of coping with environmental problems. Policy-makers and general publics are mostly accustomed to national and, in the European Union, regional governance. Functioning regulatory systems operate at these scales, but it is not clear whether these systems of governance can be successfully aligned to global goals. With respect to scale, perhaps the greatest challenge arises because real human experience of environmental change is normally felt locally, and global changes, especially those of climate, are essentially perceived as unreal because they are represented as statistical averages (Hulme, 2010), or as modelled future scenarios, with the latter often failing to present information about the locally or regionally most relevant climatic factors (e.g., precipitation and extremes).

Temporal scale. In addition to the issue of spatial scale, mismatches in time scale also pose particular challenges. There is a temporal discrepancy between the perceived occurrence of GEC and its eventual consequences, and the time-horizons of political processes. The result is a lack of urgency about identifying policy solutions. This is amplified by the time lag likely to be encountered between policy action and mitigative responses, which means that it is difficult to demonstrate benign policy effects across such scale differences. The inter-generational aspects of global change also raise questions about the attribution of the costs and benefits of action.

Complexity, disciplines, ethics and value systems. Given these pervasive scale-related problems and the inherent complexity they create, it is not surprising that inter-disciplinary and trans-disciplinary research are both seen as necessary; the problems of global change transcend conventional disciplinary enquiry. Global change is often treated largely as an environmental problem, but the environment is not simply an "independent variable"; indeed, global change is a consequence of social processes. However, given a lack of universally binding ethics and values, place-based and culturally sensitive trans-disciplinary research is needed to allow integrated study of pertinent social and natural process and their respective consequences for each other. This prioritises renegotiation of the relationship between the social and environmental sciences that can foster new research agendas suited to the policy requirements for dealing with and adapting to global change.

Uncertainty. Multi-level uncertainty pervades GEC processes, their representation in models, and the sustainable policies they require. Inter-disciplinary integrated assessment modelling has enriched exploration of uncertainty, through classifications of its multiple sources (Lempert, 2002; Walker et al., 2003). Uncertainty pertains to data and models, about both the past and possible futures generated by models, but it goes beyond this to include system identification, conceptual and computational representations, boundary and initial conditions and parameterisations. However, environmental systems may be inherently indeterminate, and we may even be ignorant about many relevant processes (Wynne, 1992). These are sources of uncertainty that cannot simply be incorporated into conventional quantitative estimates, although this is often assumed to be acceptable in order to regularise these awkward unknowns (Shackley and Wynne, 1996). There of course remains an even deeper level of uncertainty about how human values and ethics may relate to human behaviour and to human responses to global change.

As Stirling (2010) argues, we should "keep it complex", employ multiple methods, and accept ambiguity, pluralism and conditionality when providing scientific evidence to inform policy about such inherently complex and uncertain phenomena, for this helps to clarify the accountability for decision-making. Furthermore, treating GEC as a complex adaptive system will bring concepts such as co-evolution, selforganisation and emergence into the discourse, and draw on the skills of complex systems analysis, especially in the management of transitions over multiple time and space scales (Loorbach, 2007, p. 54-55; Pahl-Wostl, 2007, 2009).

\subsubsection{The need for innovation}

To meet the challenges outlined above there need to be incentives for trans-disciplinary working that can lead to pluralistic framing of problems and the co-production of knowledge between science and policy communities (implying more emphasis on a form of Mode II science; Gibbons et al., 1994). This could involve new, open-ended forms of science funding that support trans-disciplinary groups of scientists and stakeholders whose aim is to identify agreed research agendas (for example, the seed-corn, sometimes referred to as 
"sand-pit", funding of the joint Research Council programme in the UK on Rural Economy and Land Use). Results from this programme have revealed that successful inter-disciplinary working requires acceptance of the need to provide opportunities for scientists to learn one another's languages, with "translation" being a prerequisite for the development of shared research agendas (Bracken and Oughton, 2006).

Furthermore given the nature of the global change problem more methodologies for integrating different kinds of knowledge derived from different kinds of data (e.g., direct measurements such as precipitation records, historical data, and local experiences) are needed. The paradigm of the largescale simulation model is quite limited in this respect.

\subsubsection{Barriers to innovation}

The claims for innovation are not novel. However, for these innovations to occur there are several barriers to be overcome. There remain powerful forces sustaining a research focus on the paradigmatic scientific role of climate and climate change, although as noted above, global change is "felt" through real phenomena that are local (such as drought) rather than being understood through the statistics of global climate; and there are other global challenges that capture public imagination, such as biodiversity decline. There also remain deeply embedded assumptions that physical-numerical, computational models constitute a core technology to support policy, and that quantitative data are to be prioritised relative to qualitative evidence, information and "value-laden" judgement. Incorporating human values, environmental ethics and social justice into the conventional paradigm for analysis requires a radical adjustment of world-view and scientific method. There are also key assumptions about the relationship between science and policy. A belief that "science speaks truth to power" remains embedded despite the evidence of a much more nuanced, convoluted, and globally diverse set of relationships (Sutherland et al., 2012).

There is also a barrier in that data availability is not always secure, and is quite uneven regarding global coverage. Large areas of the Earth lack the necessary density of data coverage even for reliable modelling by conventional methods (notably but not exclusively in Africa). Even in economically wealthy countries, this density may be threatened by short-term policy exigencies. Another known problem is related to intertemporal comparability, continuity and reliability in data streams versus short-term innovation and instability, especially when innovation is prioritised in research funding. Typical examples can be found, for example, in remotely sensed environmental data, but also in social science data, where long-term monitoring has been less systematic and funding for data collection is limited.

\subsection{Transition to sustainable resource governance and management}

3.2.1. What do we know about sustainability transitions and can they be managed?

There is broad agreement in scientific and policy communities that sustainable development in the face of global change requires major structural adjustment at the system level (Geels, 2002; Smith et al., 2005; Pahl-Wostl, 2009). Production and consumption systems, resource governance and management regimes must all undergo fundamental change. Hence, increasing attention has been devoted to understanding the dynamics of regime transitions and the potential and limitations of managing them. Two research streams have developed, largely independently from each other - one focusing on socio-technical transitions and the other on social-ecological systems and regime transformations (Smith and Stirling, 2008). Both of these use the concept of complex adaptive systems, emphasize evolutionary dynamics of regime change, and advocate flexible, learning-oriented approaches. This reflects a shift from a mainly goal-oriented to a process-oriented emphasis in sustainable development policy. It also reflects a shift from a technocratic control paradigm to an explicit recognition of complexity and the limits of purposeful design and steering of sustainability transformations.

Managing transitions (Pahl-Wostl, 2007, 2009; Rotmans et al., 2001; Geels, 2002) is less of a purposeful process, but is instead characterized by processes of self-organization and emergence. Transition Management has established itself as a new methodology based on the multi-level, multi-phase conceptualisation of transitions (Geels, 2002). It aims to provide an integrative framework within which existing as well as new instruments are interlinked and implemented, based on a transition process analysis. The basic hypothesis is that the dynamics of transitions provide the basis for developing strategic actions that in turn influence these dynamics; this reflexivity is the hallmark of understanding and managing transitions as evolutionary processes. PahlWostl (2009) developed a comprehensive conceptual framework for how such evolutionary change can be translated into changes in different dimensions of the governance system, such as institutions, multi-level interactions or actor networks.

Methods increasingly being used and further developed for the specific needs of transition management are scenario planning, backcasting, embedded case studies, and group model building (Scholz, 2011). These methods embrace analytical and intervention dimensions within a transdisciplinary process. They aim to cross the divide between an "external representation" of reality and a social constructivist perspective, which remains a considerable challenge (Pahl-Wostl, 2007).

\subsubsection{Barriers to system transitions and major knowledge}

gaps

Systems have undergone path-dependent evolution over long periods, and the mutual interdependence of system elements stabilize current regimes, forming a major reason for resistance to change. Values and beliefs are also stabilized by the logic of the reigning paradigm embedded in many regime elements (e.g. organizations, formal regulations, professional practices, daily routines, technical infrastructure).

Little empirical knowledge exists of these stabilization phenomena, and what does exist is fragmented, with case study results often not being comparable due to a lack of shared concepts and terminology. This emerged as an issue in our interviews, where "the unwillingness or inability of social sciences to do rigorous comparative case study work" was identified 
as a major barrier to building the knowledge base urgently needed. However, initial efforts are beginning to develop the necessary frameworks. Ostrom (2007) suggested organizing variables of interest in the study of social-ecological system in a nested, multi-tiered framework. Furthermore, a management and transition framework has been developed by PahlWostl et al. $(2007,2010)$ to analyse multi-level water governance and management regimes. Both approaches provide a shared language without imposing a theoretical framework. They provide a flexible capability to enable analysts to tailor their enquiries according to the needs of the issues under consideration; and both rely on a systemic perspective that embraces complexity in a comprehensive representation. One may argue that there is a tension between the claim for pluralism and the claim for shared concepts and terminology. Pluralism results in fragmentation when it is impossible to communicate about similarities and differences of approaches chosen. This prevents progress and in particular does not support the integration of complementary perspectives to develop a more complete and rich understanding of the complexity of phenomena under consideration.

\subsubsection{Priorities for innovation and the needs for action}

A better understanding is required of the roles of both structural factors and agents, and their mutual interdependence. Few, if any, conceptual or exploratory simulation models exist that capture the complexity of transitions and permit analysis of both the potential and the limitations of managing them (Haxeltine et al., 2008; Holtz, 2010).

In several of our interviews the potential of analysing historical examples was highlighted. Such studies may provide insights into those factors that influence the (lack of ability of societies to respond to changes that undermine their long-term survival. Loorbach et al. (2011) summarized a range of research questions from a transition research perspective on governance for sustainability.

Insights from interviews and the discourse in the scientific literature translated into the following calls for action:

- To develop better tools and methods for monitoring global change and supporting anticipatory, reflexive and adaptive societal responses, and for embedding these in their societal context (evolutionary perspectives on change).

- To develop case study methodologies that allow for comparison across a wide range of cases. Data, information and knowledge bases all need to be considered and developed as common-pool resources forming a foundation for wide ranging comparative case study analyses.

- To improve access to and comparability of large data sets, including long-term support for the maintenance and further development of infrastructure and better metadata.

- To support collaborations for developing and testing integrated methodologies, where experimentation with different approaches is encouraged. Viable methodologies that generate interdisciplinary knowledge are not developed on paper, but in practice. This requires greater freedom in funding strategies.

- To support large scale and long-term comparative efforts on emerging topics of societal concern, leading to shared data and knowledge as products, allowing sufficient time for participants in these processes to capitalize on the capacity for interdisciplinary work, and to develop fruitful exchange across the science-policy interface.

- To support a network of long-term studies, including scoping and preparation phases to build the capacity for co-production of knowledge among the different academic fields involved and across the science-policy interface.

\subsection{The role of human agency in developing adaptive responses}

\subsubsection{What do we know about human agency?}

This third question focused on the role of human agency in developing adaptive responses to global change in socialecological systems, whose core covers the roles of individuals in society and in their relationships with the environment. Human agency expresses the actions and choices made by human beings (as individuals, groups, and institutions) in their interactions with their environment, and the consequent capacities to change the course of events. As pointed out by Dellas et al. (2011) those capacities should derive from authority and not simply just from power or brute force and, in that, the relevance of GEC governance emerges.

Anthropogenic climate change (and other global change phenomena, such as biodiversity loss) can thus be considered as an emergent signal resulting from the activities of a multiplicity of agents and their interactions with global ecosystems. For example, demand for energy based on fossil fuel emerges from individual consumption patterns, which are influenced by a host of cultural, market, historic, climatic and other factors. Indeed, it is only through the analysis of the behaviours of individuals and groups within socio-ecosystems that we can provide scientifically sound methods for exploring and understanding the emergent properties of such complex and adaptive systems. In turn, it is only through the understanding of the emergent properties of social-ecological systems that we can develop the capabilities needed for any robust scientific approach in support to adaptation to global change.

Developing capabilities to analyse and, possibly, simulate the behaviour of individuals and groups within different societal structures and environmental contexts appears as one of the most promising avenues for understanding and managing changes. In this regard, as pointed out by Balbi and Giupponi (2010), there is an increasing awareness that global change dynamics and the related socio-economic implications involve a degree of complexity that is not captured by traditional economic approaches based on equilibrium models. In particular, such top-down analyses of human-environment systems are not able to consider the emergence of new behavioural patterns, and evidently neither are they able to upgrade current understanding in order to learn. This may eventually lead (and has in fact led in many cases) to a flawed policy-making process which relies on unrealistic assumptions (Moss et al., 2001).

Interestingly, debates within both economics and climate science promise new opportunities for more integrated approaches, at least because they share the needs for innovative approaches in several common fields, including: 
a focus on individual behaviour and decision-making processes, and how they affect the dynamics of phenomena at various scales; the integration, distribution, and communication of knowledge, especially at the interface between science and policy making; the involvement of stakeholders not only in the decision process but also as knowledge brokers in the analysis of the phenomena; and finally, the role of technological development and its effect on society and the environment. All those issues fall within the interests of sustainability science (Kates et al., 2001; Clark and Dickson, 2003).

\subsubsection{Barriers and major knowledge gaps}

Optimism about the development of inter- and transdisciplinary efforts in sustainability science should be carefully considered from a historical perspective. The academic system is currently organized much as it was in the 19th century and it is still based on a disciplinary fragmentation that was acceptable then, but is certainly not compatible with the challenges inherent in GEC. The structure of the academy, and the reward systems for its members, should therefore be considered to be one of the main barriers to the development of the innovative approaches required.

Some disciplines will also face the need for paradigm shifts. To take economics as an example, Caballero (2010) argues that macro-economic research should abandon its current focus on local maxima in a virtual world reliant on dynamic stochastic general equilibrium models, and move to what he calls a "broad exploration" mode. Developing this argument, we might argue that more generally GEC research would significantly benefit from leaving behind the traditional approaches, and move to a new "exploration mode" in which several innovative complementary models are used.

With reference to human agency, traditional modelling approaches based upon the possibility of validation could be complemented by new exploratory modes in which no chance may exist for validation, thus raising challenging new issues in the area of policy support, including those related to the question of how to provide information useful for citizens and policy makers. Although data on human population characteristics are widely collected through censuses and surveys, data on human behaviour are scarce, often involve contested interpretation, and may encounter confidentiality concerns. In addition, it should be noted that even data on population characteristics vary widely in their quality, completeness, and comparability among countries, although there are incipient efforts to address this through greater coordination among statistical agencies.

From the above, it seems that traditional disciplinary fragmentation and data issues (availability, spatial disaggregation, and confidentiality) still represent the most important barriers that need to be overcome by future research efforts.

\subsubsection{Priorities for innovation and needs for action}

Research on the cultural acceptability of socio-technological solutions for mitigation and adaptation (e.g. alternative transportation systems, changes in settlement and habitation forms, new agricultural methods) will require innovations in psychological and anthropological research methods coupled with insights from market research. Wider availability of market research data for academic research could facilitate insights (Duchin, 2003), as would the application of sociological and anthropological research methods such as surveys and participant observation.

Information and Communication Technology is increasingly facilitating participatory and consultative processes; for example, land use plans in some countries are made available to the public through the internet, thereby allowing citizens to contribute to improving their overall quality. Furthermore, Web 2.0 and citizen science are changing traditional data collection and analysis paradigms. Much behavioural information can be acquired passively, for instance through cellular phones or Internet "cookies", and also actively, with citizens contributing voluntarily to building and enhancing databases such as Open Street Map (for building improved data on roads), the EEA Water Watch (for collecting water quality data in support of implementation of the Water Framework Directive), and the Cornell Laboratory of Ornithology's Citizen Science program (for collecting bird counts and behavioural data). Although these are promising new data sources, it should be noted that "crowd sourced" data still require data stewards, whether scientists or elected from the community, for quality control.

A number of priority areas for research and action were identified in our interviews:

- When performing research including stakeholders it is important to be clear who is responsible for what and who can play which role in the future. Research therefore has to tailor its tools to the different stakeholders.

- Local communities and their differential knowledge contributions are essential in developing adequate models and strategies, and early involvement of all relevant stakeholders (at appropriate levels of 'depth') is a key to success in consolidating knowledge and anticipating possible areas of conflict.

- Standardization of socio-economic data is required, both in terms of the population characteristics collected, and their organization in formats (such as grids) that can be easily integrated with biophysical data (cf. Balk et al., 2010).

- There is a need for innovation in the social sciences to address issues related to pattern and scale, and also to bridge the divide between quantitative and qualitative social scientists.

- There is a need to move from methods and tools oriented mainly towards the representation of - often arbitrarily identified - sub-systems or sectors, to those oriented towards the holistic analysis and simulation of complex adaptive and transformative social-ecological systems.

- It is critical to make global problems tangible for people. Science needs to address the challenge of including citizens and consumers in the process of dealing with global change, and of developing tools for understanding the connections among learning, behavioural change, action and consequences.

\section{Discussion}

\subsection{Integrating environmental and human dimensions}

There has thus far been limited consideration of the human dimension in main-stream global change research, despite 
significant progress in different fields in the social sciences. This can partly be attributed to the fact that methods applied in global change research have been dominated by one form of large-scale integrated earth system models, which has led to path-dependence and a lack of methodological pluralism. Nevertheless, a vibrant but somewhat separate community of human dimensions research has developed in recent decades, for example in integrated assessment modelling, participatory sustainability assessments or system transitions. To do justice to the complexity of the societal issues to be addressed and the richness of insights coming from social science research it will be important, but not sufficient, to develop a new generation of models where processes linked to human activities are better represented. Efforts should not be limited to integrating the human dimension into existing conceptual and methodological frameworks which would largely limit change to single-loop learning. What is needed are new modes of inquiry that question basic assumptions and transcend current practices.

What does it now mean to really integrate environmental and human dimensions? The elaboration of our three initial questions in Section 3 has shown that this implies:

- Understanding the role of the human dimension in generating global change;

- Analysing the factors that influence how problems arising from global change, and their solutions, are framed differently by different actors, and how such framing is mediated to lead to a particular societal response (or lack thereof);

- Understanding the interplay between institutional factors and human agency and its translation into the barriers and drivers of societal change; and

- Analysing the potential and the limitations for steering system transformations, and the requirements for supporting a reflexive process of societal change.

Major progress has been made on most of these themes but insights are highly fragmented, and we still lack methodologies that would weave the component strands into a fabric that could serve as a foundation for robust societal action.

The most serious gaps identified are:

- Comprehensive methodologies to support a co-production of knowledge that bridges the divide between positivist and interpretative research paradigms;

- Case study methodologies that allow comparison across a wide range of cases studying social-ecological systems;

- Methodologies that support an integration across scales in space and time;

- Consistency in data generation, storage and distribution, and risks associated with interruption in temporal records of both environmental and social data.

As far as the collaboration between natural and social sciences is concerned, considerable potential for mutual learning lies in the collection and interpretation of empirical data. The social sciences could learn to appreciate shared data collection and analysis protocols which would allow the knowledge base to be developed to derive general insights into the complex role of the human dimension without resorting to simplistic panaceas and without giving up a reflexive mode of inquiry. The natural sciences could learn that adopting different perspective in the interpretation of empirical data enriches the debate and supports critical reflection of embedded tacit assumptions.

One of our interviewees suggested that, in their current global modelling mode, "scientists are messengers from the future", and argued that science is accordingly dangerously politicised; "the politics of climate change are irredeemably the politics of science." Scientific models forecast a future that governments must then build governance regimes to manage, an unprecedented politicisation of science that itself warrants analysis by moral philosophers, political scientists, and students of scientific and technical knowledge. Models in this guise are not only tools for hypothesis formulation and testing, but also become techniques of governance, albeit without democratic accountability or ethical standing. If modelling is used to investigate possible local adaptive behaviours, it will be essential this is a participatory process, and that models inform and support, rather than dominate and rule. Modelling tools, and modelling environments, need to be developed that permit stakeholders to deliberate on alternative policy instruments. Since it is unlikely that even an agent-based model can capture those dimensions of human experience that are the preserve of the humanities (creativity, innovation, values, and meaning), this participatory engagement is essential in order to retain the diversity of human cultural experience, and to criticise rule sets adopted by modellers that are inconsistent with local lived experience.

\subsection{Bridging scales}

A significant part of the global change research community has addressed environmental change mainly at a global scale, applying quite coarse-grained top-down approaches in their analysis. By contrast, other studies have adopted a regional focus and have studied regional implications of global change. These streams of research have developed rather in isolation from each other, even at times in conflict. As climate modelling improves its regional capability, and seeks to represent, for example, hydrology and water resources more directly, opportunities will develop for more integrated approaches. However, the challenge of the spatial misfit (Moss, 2004) between the natural units for environmental and socio-economic analysis will remain.

As noted in Section 3, many case study analyses have been conducted in isolation, and it is accordingly difficult to conduct comparative analyses and develop general insights. Some scholars have suggested what can be called a diagnostic approach, taking into account complexity in a systematic fashion (Ostrom, 2007; Young, 2008; Pahl-Wostl, 2009). Such an approach should support context sensitive analysis without being case-specific and non-transferable. This is a major methodological challenge since active stakeholder involvement is tending to drive case studies towards becoming entirely case specific. A requirement for comparative analysis would be to develop shared methodological approaches and data collection protocols that are both sufficiently formalized 
that provide the basis for comparative analyses, yet sufficiently flexible to address case-specific issues and requirements.

\subsection{Data and knowledge for global change research}

It must be recognized that science (and methodological) innovation is in part data driven. New sources of data, such as crowd sourced and citizen science data (see Section 3.3.3) and ever higher-resolution satellite imagery are driving innovations in both science and praxis (Dozier and Gail, 2009). The increasing number and sophistication of satellite instruments have led to an exponential increase in data availability to earth system scientists working on climate and biophysical systems, and have brought about significant innovations in these disciplines. Although these data can also be useful to social scientists (de Sherbinin et al., 2002; de Sherbinin, 2010), our ability to make inferences about human behaviour from satellite observations is still limited and depends heavily on field-based observations and, critically, census and survey data. Furthermore, much can be learned about socio-ecological systems and human vulnerability and resilience to GEC by integrating data from the social and natural sciences in a spatial framework (e.g., Balk et al., 2005; de Sherbinin, 2009; Dilley et al., 2005). This speaks to the continuing need to invest in traditional social science data through census taking (or registries) and survey research, and to make these data available without restriction, in spatial formats at the highest resolution possible (without violating confidentiality), and at low cost to the user. Simultaneously, there is a need to maintain continuity in satellite data streams, which may require transferring responsibility for key satellite systems (e.g., Landsat, SPOT, and MODIS) from scientific to operational agencies.

Data needs will increasingly be driven by the need for solutions to the risks brought about by global change processes. Data systems that do not contribute to praxis will be increasingly difficult to justify in a resource-constrained world in which risks from environmental, economic and social spheres appear to be multiplying. Thus, data collection and analysis supporting an understanding of human behaviour and action (as discussed in Section 3.3) will need to support policy development. For example, it is hard to imagine a transition to sustainable greenhouse gas emissions at $80 \%$ below current emissions (Allison et al., 2009) that will not involve heavy government intervention with regards to subsidies, investments, direct regulation, and tax policy. Thus data from focus groups, surveys, observations of individual and household behaviour will need to be developed in order to support such policies.

It is a crucial scientific challenge to build the capacity for developing knowledge bases that allow drawing general yet context-sensitive insights from a wide range of case studies. At present, few shared databases and protocols exist in global change research, particularly in the social sciences. In order to analyse more broadly the potential and limitations of developing shared databases and research protocols more funding is required for infrastructure development and incentives need to be generated to participate in such joint efforts.

\subsection{Overcoming structural constraints to make global change research interdisciplinary and policy relevant}

The challenges identified are not entirely novel. This suggests persistent barriers to innovation which prevent creative potential to thrive. The current incentive structure in science supports short-term optimization of individual performance rather than long-term cooperation in teams and networks with shared products. This proves to be a general problem for the scientific community but is particularly detrimental for global change research. The overhead involved in constructing the trans-disciplinary teams needed to frame and address global change questions appropriately imposes hidden costs that discourage proposals. This may be reinforced by lack of confidence in the assessment mechanisms of funding agencies to handle inter-disciplinary applications, and the perceived high risk-return ratio. Furthermore the concept of triple-loop learning posits that structural change is an evolutionary process where new modes of inquiry emerge from shared practice. This requires freedom for experimentation which is in contrast to funding practice.

The lack of long-term funding also leads to a lack of continuity for the development of methodologies and methods, of data and knowledge bases. Pragmatic solutions to cope with fragmented and short-term research efforts could come from the introduction of contractual obligations facilitating exploitation and re-use of research outcomes, including standards for the open access to data, storage formats, and maintenance over time. However, given the huge uncertainties associated with global change it is unclear what kind of knowledge will prove to be the most relevant for dealing with scientific and policy challenges; the structure of the scientific research community needs to be able to respond to these challenges. It must be flexible and responsive to emerging insights. This argues in favour of flexible network structures. At the same time research must be able to engage in long-term research activities and cooperation structures that allow the exploring and assessing of innovative research themes, and the building of capacity for effective inter-disciplinary cooperation. Hence a key task for science policy is to develop the right instruments that support these kinds of selforganizing network structures.

\section{Conclusions}

To overcome the challenges identified by our analyses, the following recommendations are made that address specific structural steps (rather than at this stage identifying specific research objectives) to be undertaken over the next five years.

(i) Research programmes should be implemented that fund networks of long-term (at least decadal) studies that analyse and build the capacity of regions to deal with GEC and its unexpected developments. Such research will need to employ methodologies that embrace both scientific analysis and engaged research, to produce actionable knowledge. Case study networks could be structured around a coordinating module that supports the development and emergence of shared conceptual and methodological frameworks, and the establishment of a shared knowledge base. Without such 
integrative frameworks the risk is high that the whole will be no more, or even less, than the sum of its parts. Fragmentation and potential disputes about contradictory results may jeopardize the constructive dialogue needed to develop insights into complexity and context dependence, and to support mutual learning. Such programmes would aim at a flexible modular structure which avoids both the organizational overhead of mega-projects, and the patchwork character of bundles of individual studies.

(ii) Research programmes should support collaborations for developing and testing integrated methodologies, where experimentation with different approaches is encouraged. Viable methodologies that generate interdisciplinary knowledge and integrate different knowledge cultures are not developed in the abstract, but in practice. Such development should take place in a network (as outlined in (i)) where mutual learning is supported and criteria are developed to evaluate and compare methods and methodologies. Again, carefully designed networks of representative case studies endorsed and supported by international funding bodies, with adequate requirements and standards for data sharing and maintenance over long term would represent an asset of fundamental relevance for future developments in the science of GEC.

(iii) Such programmes will need to develop and strongly promote their own standards of "excellence". Academic systems should demonstrate more willingness to facilitate such processes, by adapting the evaluation systems (e.g. peer reviewing, ranking criteria, and methods for the assessment of performances of individual scholars and organizations) to a context in which narrow, short-term, and discipline-based metrics of relevance, quality, and impact are no longer acceptable and meaningful. Although this may seem to imply a top-down imposition of change, these "rules of the game" are ultimately likely to be changed by as much by the emergence of actor networks that develops new codes of conduct and practice, in a bottom-up response to needs.

\section{Acknowledgements}

We acknowledge the valuable contributions of further members of the RESCUE working group Alex Haxeltine, Christoph Külls, Dale Rothman, of participants of an expert workshop, and of all the experts who were willing to be interviewed, and respondents to the online survey.

\section{REFEREN C ES}

Allison, I., Bindoff, N., Bindschadler, R., et al., 2009. The Copenhagen Diagnosis: Updating the World on the Latest Climate Science. University of New South Wales Climate Research Centre, Australia, Sydney.

Amin, A., Thrift, N. (Eds.), 1994. Globalization, Institutions, and Regional Development in Europe. Oxford University Press, Oxford.

Balbi, S., Giupponi, C., 2010. Agent-based modelling of socioecosystems: a methodology for the analysis of adaptation to climate change. International Journal of Agent Technologies and Systems 2 (4), 17-38.
Balk, D., Yetman, G., de Sherbinin, A., 2010. Construction of gridded population and poverty data sets from different data sources. In: Proceedings of the GeoStat Conference, 5-7 October 2010, Tallinn, Estonia.

Balk, D., Storeygard, A., Levy, M., Askell, J., Sharma, M., Flo, R., 2005. Child hunger in the developing world: an analysis of environmental and social correlates. Food Policy 30 (5-6), 584-611.

Bracken, L.J., Oughton, E.A., 2006. 'What do you mean?' The importance of language in developing interdisciplinary research. Transactions of the Institute of British Geographers 31, 371-382.

Caballero, R.J., 2010. Macroeconomics after the Crisis: Time to Deal with the Pretence-of-Knowledge Syndrome. MIT Department of Economics Working Paper No. 10-16. SSRN: http://ssrn.com/abstract=1683617 (27.09.10).

Clark, W.C., Dickson, N.M., 2003. Sustainability science: the emerging research program. Proceedings of the National Academy of Sciences of the United States of America 100 (14), 8059-8061.

Davenport, T.H., Prusack, L., 1998. Working Knowledge: How Organizations Manage What They Know. Harvard Business School Press, Boston, p. 5.

de Sherbinin, A., 2010. Summary of the Population Environment Research Network Cyberseminar: What are the Remote Sensing Data Needs of the Population Environment Research Community?, Available at http:// www.populationenvironmentresearch.org/papers/ RS_cyberseminar_summary.pdf.

de Sherbinin, A., 2009. The Biophysical and Geographical Correlates of Child Malnutrition in Africa. Population, Space and Place Vol. 15, Available at http://dx.doi.org/10.1002/ psp.599.

de Sherbinin, A., Balk, D., Yaeger, K., Jaiteh, M., Pozzi, F., Giri, C., 2002. Social Science Applications of Remote Sensing. A CIESIN Thematic Guide. Center for International Earth Science Information Network of Columbia University, Palisades, NY.

Dellas, E., Pattberg, P., Betsil, M., 2011. Agency in earth system governance: refining a research agenda. International Environmental Agreements 11, 85-98.

Dilley, M., Chen, R., Deichmann, U., Lerner-Lam, A., Arnold, M., 2005. Natural Disaster Hotspots: A Global Risk Analysis. Analysis produced for the World Bank, Available at http:// sedac.ciesin.columbia.edu/hazards/hotspots/ synthesisreport.pdf.

Dozier, J., Gail, W.B., 2009. The Emerging science of environmental applications. In: Hey, T., Tansley, S., Tolle, K. (Eds.), The Fourth Paradigm: Data-Intensive Scientific Discovery. Microsoft Research, Redlands, WA.

Duchin, F., 2003. Household lifestyles: ideas for a research program. In: Paper Prepared for a Workshop Sponsored by the Population-Environment Research Network, 19 October 2003, Montréal.

Geels, F.W., 2002. Technological transitions as evolutionary reconfiguration processes: a multi-level perspective and a case-study. Research Policy 31 (8-9), 1257-1274.

Gibbons, M., Limoges, C., Nowotny, H., Schwartzman, S., Scott, P., Trow, M., 1994. The New Production of Knowledge. The Dynamics of Science and Research in Contemporary Societies. Sage, London.

Hackmann, H., St. Clair, A.L., 2012. Transformative Cornerstones of Social Science Research for Global Change. International Social Science Council, Paris., Available atIn: http://www.worldsocialscience.org/pdf/ ISSC_Transformative_Cornerstones_Report.pdf.

Haxeltine, A., Whitmarsh, L., Bergman, N., Rotmans, J., Schilperoord, M., Köhler, J., 2008. A conceptual framework 
for transition modelling. International Journal of Innovation and Sustainable Development 3, 93-114.

Holtz, G., 2010. Modelling system innovations in coupled human-technology-environment systems, PhD Thesis, Institute of Environmental Systems Research, University of Osnabrück, Germany.

Hulme, M.J., 2010. Problems with making and governing global kinds of knowledge. Global Environmental Change 20, 558574.

ICSU (International Council of Scientific Unions), 2010. Grand Challenges in Global Sustainability Research: A Systems Approach to Research Priorities for the Decade. International Council for Science, Paris. , In: http://www.icsu.org/ publications/reports-and-reviews/grand-challenges.

Irwin, A., 1995. Citizen Science. Routledge, London.

Kates, R., Clark, W., Corell, R., Hall, J., Jaeger, C., Lowe, I., McCarthy, J., Schellnhuber, H.-J., Bolin, B., Dickson, N., Faucheux, S., Gallopin, G., Grubler, A., Huntley, B., Jager, J., Jodha, N., Kasperson, R., Mabogunje, A., Matson, P., Mooney, H., 2001. Sustainability science. Science 292 (5517), 641-642.

Lempert, R.J., 2002. New Decision Sciences for Complex Systems. Proceedings of the National Academy of Science 99 (90003), 7309-7313.

Loorbach, D.M., 2007. Transition Management. New mode of governance for sustainable development. PhD Thesis, Rotterdam University. International Books, Utrecht, the Netherlands.

Loorbach, D.M., Frantzeskaki, N., Thissen, W., 2011. A transition research perspective on governance for sustainability. In: Jaeger, C.C. (Ed.), European Research on Sustainable Development. Springer Vlg, Berlin, Heidelberg, pp. 73-89.

Moss, S., Pahl-Wostl, C., Downing, T., 2001. Agent-based integrated assessment modelling: the example of climate change. Integrated Assessment 2 (1), 17-30.

Moss, T., 2004. The governance of land use in river basins: prospects for overcoming problems of institutional interplay with the EU Water Framework Directive. Land Use Policy 21, 85-94.

Ostrom, E., 2007. A diagnostic approach for going beyond Panaceas. Proceedings of the National Academy of Science 104, 15181-15187.

Pahl-Wostl, C., 2007. Transition towards adaptive management of water facing climate and global change. Water Resources Management 21 (1), 49-62.

Pahl-Wostl, C., 2009. A conceptual framework for analysing adaptive capacity and multi-level learning processes in resource governance regimes. Global Environmental Change 19, 354-365.

Pahl-Wostl, C., Möltgen, J., Ebenhöh, E., Holtz, G., 2007. The NeWater management and transition framework. In: PahlWostl, C., Kabat, P., Möltgen, J. (Eds.), Adaptive and Integrated Water Management. Coping with Complexity and Uncertainty. Springer Verlag, Heidelberg, Germany, pp. 75-96.

Pahl-Wostl, C., Holtz, G., Kastens, B., Knieper, C., 2010. Analysing complex water governance regimes: the management and transition framework. Environmental Science and Policy 13, 571-581.

Reid, .W.V., Bréchignac, C., Lee, Y.T., 2009. Earth system research priorities. Science 325, 245.

Rowley, J., 2007. The wisdom hierarchy: representations of the DIKW hierarchy. Journal of Information Science 33 (2), 163180 doi:10. 1177/0165551506070706.

Rotmans, J., Kemp, R., van Asselt, M., 2001. More evolution than revolution: transition management in public policy. Foresight 3 (1), 15-31.

Schellnhuber, H.J., 1999. 'Earth System' analysis and the second Copernican revolution. Nature 402, C19-C23.
Scholz, R.W., 2011. Environmental Literacy In Science and Society: From Knowledge To Decisions. Cambridge University Press, New York.

Shackley, S., Wynne, B., 1996. Representing uncertainty in global climate change science and policy: boundary-ordering devices and authority. Science, Technology and Human Values 21 (3), 275-302.

Smith, A., Stirling, A., Berkhout, F., 2005. The governance of sustainable sociotechnical transitions. Research Policy 34, 1491-1510.

Smith, A., Stirling, A., 2008. Social-Ecological Resilience and Socio-Technical Transitions: Critical Issues For Sustainability Governance. STEPS Working Paper 8. STEPS Centre, Brighton.

Sprinz, D.F., 2009. Long-term environmental policy: definition, knowledge, future research. Global Environmental Politics 9 (3), 1-8.

Stirling, A., 2010. Keep it complex. Nature 468, 1029-1031.

Sutherland, W.J., et al., 2012. A collaboratively-derived sciencepolicy research agenda. PLoS ONE 7 (3), e31824 doi:10.1371/ journal.pone.0031824.

Underdal, A., 2008. Determining the causal significance of institutions: accomplishments and challenges. In: Young, O.R., King, L.A., Schroeder, H. (Eds.), Institutions and Environmental Change: Principal Findings, Applications, and Research Frontiers. MIT Press, Cambridge, MA, pp. 49-78.

Walker, W., Harremoes, P., Rotmans, J., van der Sluijs, J., van Asselt, M., Janssen, P., Krayer von Krauss, M.P., 2003. Defining uncertainty: a conceptual basis for uncertainty management in model-based decision support. Integrated Assessment 4 (1), 5-18.

Wallace, D.P., 2007. Knowledge Management: Historical and Cross-Disciplinary Themes. Libraries Unlimited, Santa Barbara, California, United States, pp. 1-14.

Webster, A., 2007. Crossing boundaries: social science in the policy room. Science, Technology and Human Values 32 (4), 458-478.

Wynne, B., 1992. Uncertainty and environmental learning: reconceiving science and policy in the preventive paradigm. Global Environmental Change 2, 111-127.

Young, O.R., 2008. Building regimes for socio-ecological systems: institutional diagnostics. In: Young, O., King, L., Schroeder, H. (Eds.), Institutions and Environmental Change. MIT Press, Cambridge, pp. 115-144.

Claudia Pahl-Wostl is professor for resource management and Director of the Institute of Environmental Systems Research at University of Osnabrück, Germany. She is an internationally wellknown expert in adaptive management, water governance, participatory integrated assessment and modelling. Claudia PahlWostl has participated in numerous EU projects and has been the coordinator of the EU IP NeWater (New approaches for adaptive water management under uncertainty). She has numerous contributions to the scientific literature on adaptive water management, participatory integrated assessment, social learning and water governance.

Carlo Giupponi is professor at the Department of Economics and Director of the Inter-university Doctoral School ChangeS on Global Change Science and Policy of the University of Venice (Italy). Teaching activities are focused on ecological economics, integrated modelling and evaluation methods. Research activities are targeted to decision support and science-policy interface in the fields of natural resources management and climate change adaptation. He is affiliate scientist at the Euro-Mediterranean Centre for Climate Change (CMCC), and at the FondazioneEni Enrico Mattei (FEEM), Water topic leader. 
Keith Richards is professor at the Department of Geography at the University of Cambridge He has been Head of Department, Director of the Scott Polar Research Institute and Vice-President (Research) of the Royal Geographical Society-Institute of British Geographers. His research interests are wide ranging, from fluvial geomorphology and hydrology, through water resource management and its institutions, to the development of environmental research methods and agendas, and the relationship of environmental science and policy.

Claudia Binder holds a PhD in environmental sciences and a venia legendi in Human- Environment Systems from the Swiss Federal Institute of Technology, Zürich. Since 2011 she is full professor for Human Environment Relations at the Department of Geography at the University of Munich.She held positions as professor for Social and Industrial Ecology at the University of Zurich and professor for Systems Sciences at the University of Graz, Austria. Her research interests lie in analyzing and modeling transitions towards sustainability. Key research areas are sustainable resource management, regional development and value added chains.

Alex de Sherbinin is a senior researcher at the Center for International Earth Science Information Network (CIESIN), a research and data center of the Earth Institute at Columbia University. He is deputy manager of the NASA Socioeconomic Data and Applications Center (SEDAC) operated by CIESIN and coordinator of the
Population-Environment Research Network (PERN) of the International Union for the Scientific Study of Population (IUSSP) and the International Human Dimensions Programme. Mr. de Sherbinin is a geographer whose research interests focus on the human dimensions of global environmental change from local to global scales.

Detlef Sprinz is Senior Scientist at the Departmentof Transdisciplinary Concepts \& Methods at the Potsdam Institute for Climate Impact Research and honorary professor at the Department of Economic and Social Sciences, University of Potsdam, Potsdam, Germany.

Theo Toonen is Dean of the Faculty Technology, Policy and Management of Delft University of Technology. He is Chair in Institutional Governance and Public Administration at Delft University of Technology and Leiden University.

Caroline van Bers has worked predominantly with academic institutes initiating and managing interdisciplinary, applied research initiatives, as well education and capacity development activities in the fields of sustainable development, integrated assessment, adaptive water management and food systems. She now manages a small, but internationally-active environmental consultancy based in Germany, seeconsult $\mathrm{GmbH}$. Until recently, she also coordinated the activities of The Integrated Assessment Society (TIAS). 